\title{
Innovación didáctica para la comprensión del concepto de vacío en hidrostática
}

\section{Didactic innovation for the understanding of the concept of vacuum in hydrostatics}

\author{
Franklin Molina-Jiménez \\ Universidad Central del Ecuador, Quito, Ecuador \\ femolina@uce.edu.ec \\ https://orcid.org/0000-0002-2374-2192
}

\author{
Lucia Goyes-Chulde \\ Unidad Educativa Municipal Calderón, Quito, Ecuador \\ uemc.lgoyes@gmail.com \\ https://orcid.org/0000-0001-7806-5312
}

(Recibido: 11/03/2020; Aceptado: 15/03/2020 Versión final recibida: 18/04/2020)

Cita del artículo: Molina-Jiménez, F. y Goyes-Chulde, L. (2020). Innovación didáctica para la comprensión del concepto de vacío en hidrostática. Revista Cátedra, 3(2), 112-125.

\section{Resumen}

El presente artículo describe una propuesta de innovación didáctica dirigida a mejorar la comprensión del concepto de vacío, que es estudiado en la hidrostática rama de la física y constituye uno de los temas más complejos e importantes de comprender, ya que está involucrado en muchas actividades que el ser humano desarrolla, desde el proceso fundamental de respirar, hasta el de generar numerosos avances científicos e industriales.

La ausencia total de la materia en un determinado lugar recibe el nombre de vacío, ha sido analizada de varias formas, principalmente de forma experimental, sin embargo, la presente propuesta está dirigida a estudiarla desde el punto de vista teórica y comprobar su existencia en forma práctica, a través de la aplicación de la técnica didáctica de la pregunta respuesta y de la estrategia grupal investigación en el laboratorio respectivamente.

La investigación se desarrolló bajo un proceso de índole cuantitativo, cuasi experimental, inductivo en la que intervinieron treinta y tres estudiantes de la Carrera de Pedagogía de las Ciencias Experimentales, Matemática y Física, quienes trabajaron en dos momentos, en 
los que se estableció que el porcentaje promedio de comprensión del tema analizados en el pretest y postest se incrementó del $44.70 \%$ al $79.55 \%$. Esto permitió intuir que el empleo de la propuesta de innovación didáctica, basada en la aplicación de la técnica didáctica preguntas respuestas y de la estrategia investigación de laboratorio conllevó a mejorar la comprensión del concepto de vacío, además de verificar cómo influye este en el quehacer diario.

\section{Palabras clave}

Comprensión, didáctica, estrategia, innovación, técnica, vacío.

\section{Abstract}

This article describes a proposal of didactic innovation aimed at improving the understanding of the concept of vacuum. The concept of vacuum that is studied in the hydrostatic branch of physics, constitutes one of the most complex and important topics to understand, since it is involved in many activities that the human being develops, from the fundamental process of breathing, to the one of generating numerous scientific and industrial advances.

The total absence of matter in a given place is called a vacuum, and it has been analyzed in several ways, mainly in an experimental way. However, the present proposal is aimed at studying it from a theoretical point of view and verifying its existence in a practical way, through the application of the didactic technique of the question-answer and the group research strategy in the laboratory, respectively.

The research was developed under a quantitative, quasi-experimental, inductive process in which thirty-three students of the Pedagogy of Experimental Sciences, Mathematics and Physics Career took part. They worked in two moments, in which it was established that the average percentage of understanding of the subject analyzed in the pre-test and post-test increased from $44.70 \%$ to $79.55 \%$, allowing us to intuit that the use of the didactic innovation proposal, based on the application of the didactic technique questions and answers and the laboratory research strategy, led to a better understanding of the concept of vacuum, in addition to verifying how it influences our daily work.

\section{Keywords}

Understanding, didactics, strategy, innovation, technique, emptiness.

\section{Introducción}

La física al ser una ciencia experimental que estudia todos los fenómenos que rigen a la naturaleza, ocasiona que el aprendizaje y comprensión de esta rama del conocimiento humano no se forje de una menara ágil en la estructura cognitiva de los estudiantes que cursan los diversos niveles académicos donde se enseña esta ciencia, además la naturaleza de la ciencia y el desarrollo del conocimiento científico influye significativamente en la forma como se enseña, viéndose reflejado en los métodos que utiliza el docente, en su actuación y toma de decisiones en el aula de ciencias naturales, así lo señala Rua y Alzate (2012), es decir que estas nociones crean la necesidad de buscar estrategias didácticas que permitan mejorar la comprensión y análisis de estos fenómenos que son estudiados en las aulas de la Carrera de Pedagogía de las Ciencias Experimentales Matemática y Física y luego 
comprobados y verificados en el laboratorio de la Unidad de Física de la Universidad Central del Ecuador, por los estudiantes en los cursos regulares.

El presente trabajo de investigación presenta una propuesta didáctica innovadora que busca mejorar la comprensión del concepto de vacío, el cual es analizado en la hidrostática, rama de la hidráulica que estudia a los fluidos en estado de equilibrio. Esta propuesta está orientada a lograr que los estudiantes puedan asimilar de manera efectiva los conceptos relacionados a ella.

La propuesta didáctica está fundamentada en el proceso de realizar preguntas a los estudiantes, ya que el arte de preguntar es el arte de seguir preguntando y esto significa que es el arte de pensar (Gadamer, 2007), lo que permitirá determinar el nivel de conocimiento adquirido por ellos al observar la recreación del fenómeno del vacío en el laboratorio de física.

Se pretende plasmar una alternativa innovadora de enseñanza, en procura de generar la comprensión no solo del tema propuesto, sino de otros relacionados a la física, para que otros docentes que laboran en el centro de estudios donde se realiza la investigación puedan mejorar su proceso de enseñanza aprendizaje y por ende el rendimiento académicos de los estudiantes que para Cascón (2000) "constituye la demostración de sus conocimientos sobre las distintas áreas o materias, que el sistema considera necesarias y suficiente para su desarrollo como miembro activo de la sociedad" (p. 11).

Trabajos relacionados como el de Urbina 2008 y el de Sánchez 2010, son uso considerados como antecedentes de la investigación realizada. En el presente documento se realiza una reseña de la investigación ejecutada, y se analiza la definición de innovación didáctica, el nivel de comprensión de acuerdo a la taxonomía de Bloom (2013), que "constituye la capacidad de comprender o aprehender, en donde el estudiante sabe qué se le está comunicando y hace uso de los materiales o ideas que se le presentan, sin tener que relacionarlos con otros materiales o percibir la totalidad de sus implicaciones" (p.2), además del estudio del concepto de vacío y otros relacionados al este a través de la utilización de equipos del laboratorio de física que permiten determinar la diferencia de presiones entre dos medios, la ausencia de sonido en el vacío, el proceso metodológico realizado para ejecutar la investigación con su respectivo análisis estadístico de los resultados obtenidos al aplicar un pretest y postest, para finalmente presentar las conclusiones.

\section{Fundamentación Teórica}

\subsection{Innovación didáctica}

Una de las características que todo docente debe poseer, es la de estar siempre dispuesto a modificar su forma de enseñar, ya que el enseñar física está en función de los avances científicos y tecnológicos. Es decir, debe generar actividades que involucren procesos de innovación educativa, aspecto que Imbernón 1996, González y Escudero 1987 señalan como una acción o conjuntos de acciones que conllevan a cambios internos y cualitativos en el quehacer educativo de un docente en procura de mejorar la comprensión, eficiencia y efectividad en la solución de problemas generados en forma inmediata.

La innovación de acuerdo con Tejada (2012), se puede considerar como "el núcleo de una acción renovadora generada dentro del aula en la que las prácticas profesionales se modifican más si se propician procesos de autorevisión y reflexión colaborativa" (p. 6). Las renovaciones conllevan situaciones nuevas dentro de un contexto que en ocasiones pueden

Licencia Creative Commons Atribución 4.0 Internacional (CC BY 4.0)

Revista Cátedra, 3(2), pp. 112-125, mayo-agosto 2020. e-ISSN: 2631-2875

https://doi.org/10.29166/10.29166/catedra.v3i2.2072 
generar reformas en los procesos educativos. Sin embargo, no siembre estas innovaciones pueden generar reformas, estableciéndose que el docente debe innovar para renovar las estrategias didácticas utilizadas en el proceso de enseñanza con sus estudiantes, acción que lo desarrolla en el aula de clase en procura de la comprensión de los conceptos de una manera significativa.

Se debe considerar que estos aprendizajes significativos son producto de experiencias innovadoras que para Torre (1997) "pueden convertirse en la primera fase de innovaciones de mayor alcance" (p. 46). Confirmando que las innovaciones surgen de experiencias innovadoras generadas en el aula de clase.

En esta perspectiva, Capelástegui afirma que una de estas experiencias innovadoras ligada a la iniciativa generada por el docente en su procedimiento didáctico, es la de buscar cambios en la utilización de las estrategias didácticas orientadas a la transferencia de conocimientos, trabajos en grupo, trabajos colaborativos y la simulación de situaciones reales, aspectos que son considerados en la presente investigación (Capelástegui, 2003).

Los principios del aprendizaje constructivista fundamentados por Piaget 1929, Vygotsky 1930 y Ausubel 1978 establecen que el sujeto construye el conocimiento de manera activa, interactuando con el objeto de estudio. En el caso del aprendizaje de la Física este se realiza en el laboratorio. El nuevo conocimiento adquiere significado cuando se relaciona con el conocimiento previo, es decir, el concepto de vacío quedará bien fundamentado cuando el estudiante comprende las acepciones sobre hidrostática, presión atmosférica y ebullición; y es capaz de encontrar las aplicaciones que el concepto de vacío tiene en la vida cotidiana. Según Oviedo (1983), la utilización de procedimientos didácticos para obtener un determinado resultado "comprende las estrategias y técnicas" (p.24). De acuerdo con Szczurek (1989), la estrategia es el "conjunto de acciones deliberadas y arreglos organizacionales para coordinar (dirigir) el sistema de enseñanza aprendizaje" (p. 89). Estas estrategias pueden ser magistrales, cooperativas, individuales y grupales.

En esta investigación se aplica la estrategia grupal ya que enfatiza el trabajo conjunto de los estudiantes en actividades de aprendizaje cooperativo, supeditadas a la tutoría del profesor en la que su rol constituye ser un facilitador del aprendizaje, como manifiesta Bastidas 2004. La estrategia grupal con la que desarrolla todo el proceso de la investigación incluye el trabajo experimental en el laboratorio, y su importancia radica no sólo en la posibilidad de la observación y de la experimentación sobre la realidad y desarrollo de habilidades experimentales, sino también y quizás más fuertemente en la posibilidad que brindan para relacionar las teorías y modelos con la experiencia y suministrar una oportunidad para que los alumnos conozcan cómo se construye el conocimiento científico, como lo confirma Hodson (1988), quien concibe al "trabajo experimental (prácticas de laboratorio) como pilar fundamental para su enseñanza” (p. 67).

De acuerdo con Bastidas (2004), la técnica constituye una "forma particular de emplear un instrumento y/o recurso en el que se apoya la enseñanza" (p. 121). La técnica que se plantea utilizar en la investigación con el fin de mejorar el proceso de aprendizaje de física es la pregunta-respuesta, misma que permite organizar los esquemas mentales en los estudiantes para facilitar la construcción de conceptos.

Gadamer (2007) señala que el "arte de preguntar, es el arte de seguir preguntando y esto significa que es el arte de pensar" (p. 134). Esto permite afirmar que las preguntas

Licencia Creative Commons Atribución 4.0 Internacional (CC BY 4.0)

Revista Cátedra, 3(2), pp. 112-125, mayo-agosto 2020. e-ISSN: 2631-2875

https://doi.org/10.29166/10.29166/catedra.v3i2.2072 
formuladas por los científicos han permitido obtener respuestas determinantes en beneficio del desarrollo científico y buscar nuevas estrategias para mejorar la enseñanza de las ciencias.

El plantear preguntas, ya sea por el docente o por el estudiante, conlleva a desarrollar en mayor relevancia la capacidad para construir nuevos conocimientos significativos que permiten estructurar nuevos esquemas mentales como la abstracción y el razonamiento, permitiendo incrementar el acervo científico de todos los involucrados. Rojas (2009), en sus indagaciones sobre la función pedagógica de la pregunta en los procesos de aprendizaje, razonamiento, influencias cognitivas y metacognitivas en ciencias, determinó que "las preguntas contribuyen de manera sistemática a la construcción en los estudiantes de los procesos de razonamiento en Ciencia" (p. 156).

Las preguntas deben cumplir ciertas características básicas para ser planteadas. Rojas (2009), señala que las preguntas deben ser reflexivas, contextualizadas, decisoria, divergente, lingüística y deben incluir como requisitos para su formulación el uso de los signos de interrogación al principio y al final de la pregunta, así mismo debe tener sujeto, predicado y verbo (p. 45).

\subsection{La compresión}

De acuerdo a Boostrom (2005), los "verdaderos aprendizajes significativos no son generados a partir de actividades que involucren memorización, sino por el contrario por actividades que permitan ejercitar las habilidades para el procesamiento de información" (p. 45), la adquisición y desarrollo de conceptos, la toma de decisiones, la selección de alternativas, análisis, síntesis, interpretaciones, resolución de problemas y creación de nuevas ideas. En este sentido, se comprende que el docente necesita disponer de un modelo que permita discernir el hecho de cómo promover el aprendizaje significativo en los alumnos y permitir el desarrollo de estas capacidades, para lo cual las taxonomías sobre los aprendizajes constituyen los modelos explicativos de cómo progresar en el aprendizaje, y las fases que este debe tener.

Dentro de la Taxonomía Cognitiva del aprendizaje propuesto por Bloom se encuentran los niveles de conocimiento, comprensión, aplicación, análisis, síntesis y evaluación. En este sentido, se puede establecer que la comprensión constituye la capacidad de comprender o aprehender; en donde el estudiante sabe qué se le está comunicando y hace uso de los materiales o ideas que se le presentan, sin tener que relacionarlos con otros materiales o percibir la totalidad de sus implicaciones. El material requiere de un proceso de transferencia y generalización, lo que demanda una mayor capacidad de pensamiento abstracto (Bloom, 1964).

Requiere que el alumno explique las relaciones entre los datos o los principios que rigen las clasificaciones, dimensiones o arreglos en una determinada materia, conocimiento de los criterios fundamentales que rigen la evaluación de hechos o principios, y conocimientos de la metodología, principios y generalizaciones, tales como la traducción, interpretación y la extrapolación.

Finalmente, la comprensión se puede considerar como una capacidad de desempeño flexible lo que permite establecer según Perkins (2003) que "comprender es la habilidad de pensar y actuar con flexibilidad partir de lo que uno sabe" (p. 70). En este contexto la idea de comprender se da cuando el estudiante es capaz de actuar de manera flexible en tareas que involucren respuestas múltiples y desempeño de actividades orientadas a generar procesos de aprendizajes significativos.

Licencia Creative Commons Atribución 4.0 Internacional (CC BY 4.0)

Revista Cátedra, 3(2), pp. 112-125, mayo-agosto 2020. e-ISSN: 2631-2875

https://doi.org/10.29166/10.29166/catedra.v3i2.2072 


\subsection{El vacío en la hidrostática.}

Los conceptos se han creado desde que el ser humano tuvo la capacidad de abstraer, es decir, de tomar del medio donde habitamos elementos significativos e intrínsecos de cada objeto o fenómeno y de asociar esos elementos en función de semejanzas y diferencias para generar conocimiento. Como lo señala Sager (1993), los conceptos se utilizan "para estructurar el conocimiento y la percepción del mundo circundante y se vale del lenguaje para su formación y comunicación" (p. 34). Este argumento guía al proceso de la comprensión de muchos fenómenos físicos observables.

Para Ausubel (1978) los "conceptos constituyen objetos, eventos, situaciones o propiedades que poseen atributos criteriales comunes y se designan, en una cultura dada, por algún símbolo aceptado" (p. 89); de esta manera, los conceptos se relacionan entre ellos para generar una nueva estructura cognitiva.

Según Moreira la adquisición de conceptos puede ocurrir de dos formas, por formación, en la que los conceptos se adquieren a través de experiencias directas durante diferentes etapas del desarrollo del ser humano y por asimilación, en los cuales los conceptos se forman a medida que adquiere más vocabulario y se pueden establecer asociaciones en la estructura cognitiva con estas palabras y de esta manera formar una nueva estructura cognitiva (Moreira, 2010).

Tomando en cuenta lo anterior, comprender el concepto de vacío se torna un poco complejo puesto que el ser humano puede inferir el concepto de materia de una manera general a todo lo que nos rodea; es decir, la cantidad de sustancia que ocupa un lugar en el espacio y posee masa, forma, peso y volumen, por lo que es observable y medible y más aún si se considera que la Luz no tiene masa, sin embargo se puede medir su energía y su momento.

Definiciones de vacío, que han sido enunciadas por Pierre Gassendi 1592, el pionero de la ciencia, consideró a este como un medio indetectable donde podían moverse, expandirse y comprimirse los átomos. Por otra parte, Newton reconoce al vacío como un medio de inmovilidad absoluta. Christian Huygens lo define como un medio material sutil y elástico. Nicolás Malebranch habló de la unidad elemental de la materia sutil, psíquica. De igual forma, Benjamín Franklin en 1752 habló de una sustancia vibratoria que llenaba todo el espacio, pero compartido con un hipotético fluido imponderable, la electricidad. Adicionalmente, Lomonósov lo definió como un medio material que llena los intersticios interatómicos. Dalton lo consideró indispensable para la vida del átomo. Charles Cesar, sin enunciar el vacío, describe el hecho de que un gas puede desaparecer a $-273^{\circ}$. Augustín Fresnel reconocía el vacío como el soporte material de las ondas luminosas.

En las teorías de Maxwell el espacio vacío aparece como conductor de tensiones y energías responsables de las acciones electromagnéticas. En el siglo XIX, Ángelo Secchi señala al vacío como la causa de los fenómenos luminoso y eléctrico que actúa vibrando en los primeros y desplazándose en los segundos. La concepción moderna del vacío está fundamentada en base al experimento realizado por Torricelli, quien en 1644 mostró un tubo vertical de un metro de longitud cerrado en la parte superior, en el cual se elevaba $76 \mathrm{~cm}$ de mercurio en condiciones normales sobre un recipiente también lleno de mercurio, este efecto indica la presión del aire circundante, además la zona del tubo por encima del límite del mercurio está vacía, condición que permite al mercurio estar suspendido (Young, 2009). Este experimento estableció la equivalencia de la presión atmosférica con la altura que alcanza el mercurio en un tubo vaciado al que se lo denominó barómetro.

Licencia Creative Commons Atribución 4.0 Internacional (CC BY 4.0)

Revista Cátedra, 3(2), pp. 112-125, mayo-agosto 2020. e-ISSN: 2631-2875

https://doi.org/10.29166/10.29166/catedra.v3i2.2072 
Otto Von Guericke en Alemania en 1654 construyó la primera bomba de vacío para poder succionar el aire del interior de un recipiente compuesto por dos hemisferios adosados, que eran tirados por 16 caballos en dos grupos de 8 en direcciones opuestas (Young, 2009). Con este experimento mostró que cuando la esfera estaba vaciada de aire, es decir, cuando estaba al vacío, la fuerza de los caballos era incapaz de separar los hemisferios, afirmando de esta manera que la presión del aire circundante supera la fuerza realizada por los caballos y cuando el recipiente contiene aire una fuerza leve consigue despegar los hemisferios, conocidos actualmente como Los hemisferios de Magdeburgo (Young, 2009).

Einstein consideraba al vacío como la existencia de las masas eléctricas, realidad física dentro del vacío. Finalmente se puede considerar la concepción de Carl Sagan (1980) astrónomo, astrofísico y divulgador científico reconocido por sus estudios realizados sobre la estructura del universo, quien afirmó que "Los átomos son, en su mayor parte, espacio vacío. La materia se compone principalmente de nada..." (p.160).

En virtud de estas afirmaciones, se establece la necesidad e importancia de desarrollar una propuesta de innovación didáctica dirigida a establecer nuevas alternativas de trabajo tanto en el aula como en el laboratorio de física que permita la enseñanza del concepto de vacío.

\section{Metodología.}

La propuesta de innovación didáctica está orientada a ser desarrollada en dos momentos, que se describen a continuación:

El primer momento de la investigación se desarrolla dentro del aula de clase de la Carrera de Pedagogía de las Ciencias Experimentales Matemática y Física de la Facultad de Filosofía Letras y Ciencias de la Educación de la Universidad Central del Ecuador, lugar en el que el docente en base a diferentes actividades guía a los estudiantes a la comprensión de los conceptos de hidrostática, vacío, presión atmosférica, ebullición, hervir, sonido, onda mecánica, hemisferios de Magdeburgo. Los treinta y tres estudiantes orientados por el docente conocen la existencia del vacío con sus propiedades y su importancia a través de la ejecución de la estrategia magistral, conferencia del tipo discusión (Bastidas, 2004), en la que los temas que se tratan son previamente estudiados por los participantes, luego de ello, son discutidos en grupos de aprendizaje. Los participantes pueden formular preguntas al instructor o viceversa.

Se procede a explicar de forma magistral en base a cuatro actividades, cómo se crea el vacío utilizando un dispositivo llamado "bomba de vacío" y los efectos que produce sobre un globo inflado y la incidencia de este fenómeno en el ser humano. Se analiza la diferencia entre los conceptos hervir y ebullición generados en el agua, además se explica que, en el vacío, el sonido no se propaga y finalmente se establece cómo la presión ejercida por el aire está en todas las direcciones a través de un instrumento llamado hemisferios de Magdeburgo. Una vez expuestos los conceptos y ejemplos a los estudiantes, se verifica el nivel de comprensión de ellos a través de la aplicación del pretest (Cuadro 1).

El segundo momento de la investigación es ejecutado en el laboratorio del Centro de Física de la Universidad Central del Ecuador, donde el trabajo con los estudiantes es ejecutado a través de la estrategia grupal llamada investigación de laboratorio (Bastidas, 2004), la que consiste en recopilar datos en forma organizada y sistemática que prueben o refuten teorías e hipótesis, o que amplíen los conocimientos de fenómenos en situaciones controladas por todos los alumnos dentro o fuera de un laboratorio, pero siguiendo el método experimental.

Los treinta y tres estudiantes, a través de un proceso experimental y guiados por el docente, ejecutaron cuatro actividades experimentales en las que se utilizan la bomba, campana y

Licencia Creative Commons Atribución 4.0 Internacional (CC BY 4.0)

Revista Cátedra, 3(2), pp. 112-125, mayo-agosto 2020. e-ISSN: 2631-2875

https://doi.org/10.29166/10.29166/catedra.v3i2.2072 
plato de vacío, vaso de precipitación, globo, agua, celular y hemisferios de Magdeburgo, de la siguiente manera:

- La primera actividad está destinada a verificar los efectos que el vacío produce sobre un globo a medio inflar en el interior de la campana. Al ser encendida la bomba, el aire de su interior es succionado y los estudiantes observan cómo el globo aumenta de tamaño, fenómeno que permite a los estudiantes obtener las respectivas conclusiones, orientadas a determinar el grado de abstracción que posee el estudiante al momento de enfrentarse con el fenómeno físico llamado homeostasis.

- La segunda actividad propuesta determina la diferencia entre hervir y ebullición, para lo cual se coloca agua en un vaso de precipitación, que es introducido en el interior de la campana de vacío para luego ser encendida la bomba. Transcurrido unos minutos los estudiantes verifican el proceso de ebullición del agua sin incremento de temperatura.

- La tercera actividad verifica cómo el sonido, al ser una onda mecánica, necesita de un medio para propagarse, mientras que en el vacío no se propaga, para lo cual se coloca un celular encendido en el interior de la campana de vacío con el reproductor de música encendido, se extrae el aire de la campana y el sonido disminuye.

- La cuarta actividad permite verificar la existencia de la presión atmosférica y cómo esta actúa sobre todos los seres que habitan en la superficie terrestre, para lo cual se utiliza el equipo de laboratorio llamado hemisferios de Magdeburgo, que consiste en dos semiesferas unidas una contra la otra, y que al succionar el aire del interior de los hemisferios genera el vacío utilizando la bomba, quedando unidas de tal forma que es tirada de los extremos por los estudiantes y por efecto de la existencia de la presión externa estas no se separan.

El pretest y el posttest es elaborado en base a ítems de opción múltiple, en el que constan preguntas con varias opciones de respuestas, de las cuales solo una es indudablemente correcta (López et al. 2013). Estos ítems buscan la comparación y evaluación de ideas, conceptos relacionados al vacío.

Las interrogantes que se plantearon en cada uno de los ítems del pretest y postest, que permitieron dar las respuestas a la investigación se orientan en:

Para la primera actividad:

- El globo en el interior de la campana de vacío comienza a incrementar su volumen ya que la presión externa del globo comparada con la presión interna del globo es: mayor, igual, menor o nula.

- La homeostasis es una característica que los cuerpos poseen cuando la presión interna del mismo comparada con la presión externa es: igual, mayor, menor o nula.

- El globo en el interior de la campana de vacío, incrementa su volumen debido a que se crea: volúmenes grandes de aire, el vacío, masas de aire elevadas o mayor densidad de aire.

Para segunda actividad:

Licencia Creative Commons Atribución 4.0 Internacional (CC BY 4.0)

Revista Cátedra, 3(2), pp. 112-125, mayo-agosto 2020. e-ISSN: 2631-2875

https://doi.org/10.29166/10.29166/catedra.v3i2.2072 
- Las burbujas de agua que se producen en el vacío son efecto de: incremento de temperatura, decremento de temperatura, ausencia de aire o aumento del volumen de aire.

- La separación de las moléculas de agua en el interior de la campana de vacío se debe a que el agua se encuentra en proceso de: congelamiento, compresión, hervir o descompresión.

- Hervir significa que la temperatura del agua: disminuye, aumenta, se equilibra o se deteriora.

- Ebullición significa que las moléculas de agua se: unen entre sí, separan, entrelazan o golpean.

- Existe alguna diferencia entre hervir agua y la ebullición del agua: si, no, ninguna o posiblemente.

Para la tercera actividad:

- El sonido de la música que emite el celular: aumenta en intensidad, disminuye en intensidad, se mantiene la intensidad o desaparece.

- La causa de no escuchar la música en el interior de la campana de vacío es: demasiado aire en el interior, se creó una masa de aire, ausencia de aire o poco aire.

Para la cuarta actividad:

- Al comenzar a tirar los extremos de las esferas estas se separan: si, no, con facilidad o con dificultad.

- La presión que el aire ejerce alrededor de los hemisferios es: en la parte superior de estos, en la parte inferior, en todas las direcciones o en ninguna dirección.

- Al abrir la llave de ingreso de aire en los hemisferios, este: sigue ejerciendo presión, sigue ejerciendo fuerza, ingresa y se separan los hemisferios o comprime las paredes de los hemisferios.

$\mathrm{Al}$ analizar el número de respuestas correctas después de ser aplicados el pretest y postest a los estudiantes se puede determinar que ellos han sido capaces de comprender los conceptos relacionados al vacío, lo que permitiría generar procesos de aprendizajes significativos.

\section{Resultados}

Para evaluar la comprensión de lo realizado en forma experimental, los estudiantes responden las preguntas planteadas en su hoja de trabajo, constituyéndose el postest. (Cuadro 1)

\begin{tabular}{lcccc}
\hline PREGUNTA & \multicolumn{2}{c}{ PRETEST } & \multicolumn{2}{c}{ POSTEST } \\
\hline ACTIVIDAD 1 & \multicolumn{2}{c}{ EFECTO DEL VACÍO EN UN GLOBO } \\
ACIERTOS & 13 & $39.39 \%$ & 22 & $66.67 \%$ \\
DESACIERTOS & 20 & $60.61 \%$ & 11 & $33.33 \%$ \\
ACTIVIDAD 2 & DIFERENCIA ENTRE HERVIR Y EBULLICIÓN DEL AGUA \\
ACIERTOS & 15 & $46.46 \%$ & 28 & $84.85 \%$ \\
DESACIERTOS & 18 & $53.94 \%$ & 5 & $15.15 \%$ \\
ACTIVIDAD 3 & \multicolumn{3}{c}{ PROPAGACIÓN DEL SONIDO EN EL AIRE } \\
\hline
\end{tabular}

Licencia Creative Commons Atribución 4.0 Internacional (CC BY 4.0) 


\begin{tabular}{lcccc}
\hline ACIERTOS & 15 & $45.45 \%$ & 26 & $78.79 \%$ \\
DESACIERTOS & 18 & $54.55 \%$ & 7 & $21.21 \%$ \\
ACTIVIDAD 4 & & HEMISFERIOS DE MAGDEBURGO \\
ACIERTOS & 16 & $48.48 \%$ & 29 & $87.88 \%$ \\
DESACIERTOS & 17 & $51.52 \%$ & 4 & $12.12 \%$ \\
\hline
\end{tabular}

Cuadro 1. Resultados por actividad desarrollada

Los resultados obtenidos al evaluar las respuestas de los estudiantes en el proceso del pretest y postest permiten establecer información relevante con respecto a la utilización de la estrategia grupal denominada investigación en el laboratorio y de la técnica didáctica, pregunta-respuesta en el proceso de enseñanza-aprendizaje de los conceptos relacionados con el vacío y de verificar sus efectos, información que permitirá guiar a establecer una propuesta de innovación didáctica.

Los aciertos obtenidos en un rango comprendido entre 1 y 33 de los test y postest desarrollados por los estudiantes, fueron considerados en base a un número entero y su equivalente en porcentajes. Es así como los valores de aciertos al responder las preguntas de la primera actividad relacionada con el efecto del vacío sobre un globo en relación con el pretest y al postest tuvo una variación positiva del 27.28\% (Cuadro 1), permitiendo establecer que el globo en el interior de la campana incrementa su volumen debido a que se crea el vacío, y el efecto de la homeostasis que los cuerpos poseen en la superficie terrestre se puede modificar en ausencia del aire.

Para la segunda actividad, la variación es del 38.39 \% (Cuadro 1), y afirma que los estudiantes si establecen la diferencia entre los conceptos de hervir y de ebullición del agua y que existe una forma de generar la ebullición sin incremento de temperatura. La tercera actividad tiene una variación del $33.34 \%$ (Cuadro 1), en consecuencia, los estudiantes comprenden que la causa de no escuchar la música en el interior de la campana de vacío es debido a la ausencia de aire en el interior de la misma ya que el sonido es una onda mecánica. La cuarta actividad tiene una variación del $39.40 \%$ (Cuadro 1), de esta forma verifican que la presión atmosférica que actúa sobre cualquier objeto en la superficie terrestre está presente. Se debe resaltar que las preguntas relacionadas con el hemisferio de Magdeburgo son las que presentaron mayor porcentaje de variación de respuestas correctas (39.4\%).

Finalmente, el porcentaje promedio de variación comparando las cuatro actividades en conjunto corresponden al 34.85 \% (Cuadro 2), evidenciando que los estudiantes mejoraron la comprensión de las propiedades que el vacío tiene sobre los cuerpos. Estas afirmaciones se pueden visualizar de mejor manera en la Figura 1, en la que se expresa la variación de los aciertos en porcentaje del pretest y postest.

\begin{tabular}{lcccc}
\hline \multicolumn{1}{c}{ PREGUNTAS } & \multicolumn{2}{c}{ PRETEST } & \multicolumn{2}{c}{ POSTTEST } \\
\hline ACIERTOS & 14.75 & $44.70 \%$ & 26.25 & $79.55 \%$ \\
DESACIERTOS & 18.25 & $55.33 \%$ & 6.75 & $20.45 \%$ \\
\hline
\end{tabular}

Cuadro 2. Promedio de las cuatro actividades desarrolladas 


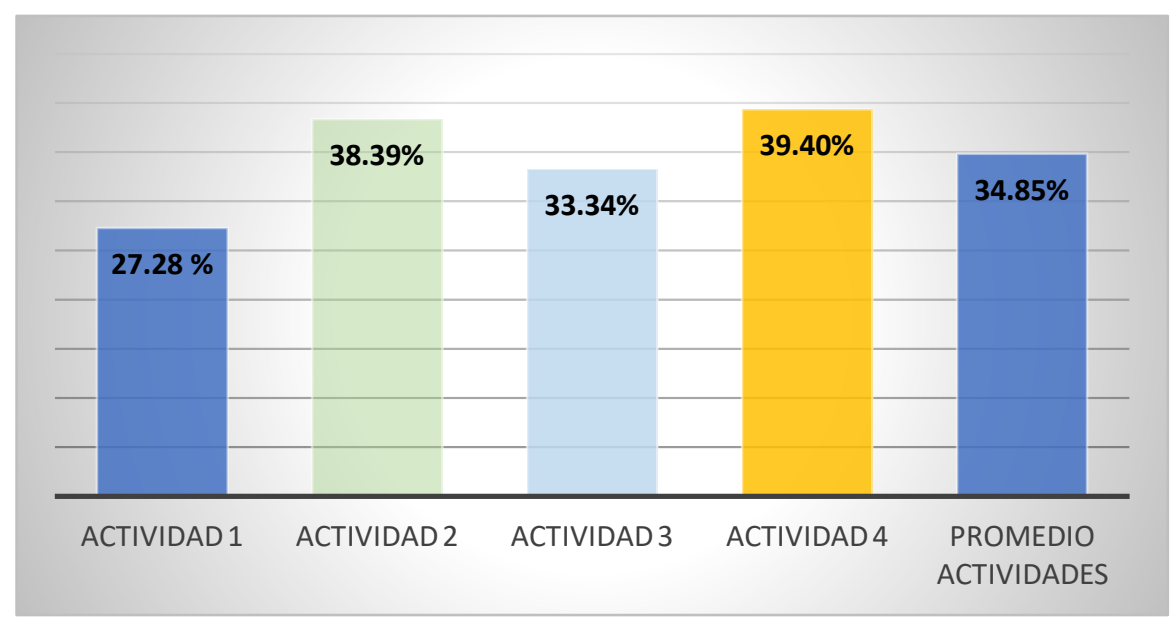

Figura 1. Variación de incremento del porcentaje de aciertos entre el pretest y postest.

\section{Discusión.}

Los resultados permiten establecer que la presente propuesta de innovación didáctica para mejorar la comprensión del concepto de vacío y verificar sus efectos, utilizando la técnica didáctica peguntas respuestas y la estrategia grupal de investigación en el laboratorio sí contribuye a mejorar el proceso de enseñanza-aprendizaje, y por ende mejoran los procesos de la comprensión de los conceptos estudiados en Física.

Para las preguntas dirigidas a los estudiantes se utilizó el formato de ítems de opción múltiple ya que según García (2006) este tipo de formato permite "evaluar una amplia gama de objetos de aprendizaje desde conocimientos factuales hasta procedimentales" (p.21). Además, esta evaluación permite ser objetiva, es decir, no admite la interpretación de la respuesta

Como lo señala Sánchez (2010) "el uso de las preguntas en el ámbito Universitario permite a los estudiantes aprender a aprender, promueve la discusión de los contenidos, mantiene una buena interacción y participación durante la clase, lo que lleva a adquirir aprendizajes significativos" (p.22). Estas afirmaciones permiten establecer que el proceso desarrollado en la presente investigación es lo suficientemente bueno para continuar aplicándolos en posteriores situaciones dentro del aula.

Como se puede observar en el Cuadro 2 y en la Figura 1, el porcentaje promedio de estudiantes que responden correctamente a las preguntas planteadas en cada una de las actividades en el primer momento ejecutadas en el aula de clase mediante el pretest y posteriormente en el segundo momento desarrolladas en el laboratorio de Física a través del postest, se incrementa del $44.70 \%$ al $79.55 \%$. De esta manera, se puede afirmar que el empleo de la propuesta didáctica basada en la investigación de laboratorio y la aplicación de preguntas dirigidas a los estudiantes conlleva a la comprensión de los conceptos relacionados con el vacío (Urbina,2008, p. 56)

Investigaciones realizadas por Urbina sobre las estrategias metodológicas para potenciar la comprensión de la Física le permitieron establecer que se debe propiciar el trabajo con la participación real y efectiva de los estudiantes en el proceso educativo, afirmación que ha sido corroborada por la presente investigación permitiendo establecer la validez de esta. 
En esta investigación, a diferencia de las realizadas por Urbina 2008 y la de Sánchez 2017 se ejecutó realizando actividades prácticas, utilizando los equipos del laboratorio de Física, haciéndolas más significativas para el aprendizaje de los conceptos; por ende, las respuestas obtenidas al contestar el postest tuvieron un mayor rango de respuestas correctas. Por tal razón, se puede considerar la factibilidad de realizar esta actividad en el aula de clase y complementarla en el laboratorio de Física con regularidad en función de los temas tratados en hidrostática.

\section{Conclusiones}

Los docentes universitarios deben desarrollar nuevas propuestas didácticas de aprendizaje que promuevan en los estudiantes un pensamiento científico, crítico y reflexivo hacia la Física, y una de ellas es que los docentes deben considerar la necesidad de plantear preguntas a sus estudiantes durante todo el proceso de enseñanza-aprendizaje llevado en el aula y en el laboratorio de física, para lograr un ambiente de constante trabajo por parte del estudiante, además esta estrategia didáctica se puede replicar en otras instituciones alineadas en el campo de la educación superior, con el fin de verificar su validez con otros grupos de estudiantes y en otros contextos.

Se puede aprender haciendo preguntas, pues contribuye a mejorar la capacidad de aprendizaje y más aún la estructura cognitiva del individuo. Para lograr este proceso, los enunciados deben ser claros y comprensibles, se debe tomar en cuenta el tiempo suficiente para la lectura, la comprensión de la pregunta y la selección de la respuesta.

Se pudo determinar que trabajo ejecutado por los estudiantes es efectivo en el proceso educativo permitiendo generar verdaderos procesos de aprendizajes significativos. Sin embargo, se debe considerar en investigaciones futuras, determinar cómo influye la utilización de la tecnología de información y la comunicación en el proceso de enseñanza aprendizaje de los conceptos relacionados al vacío.

\section{Bibliografía}

Ausubel, D. (1978). Psicología Educativa, un punto de vista cognitivo. Trillas. México.

Bastidas, P (2004). Estrategias y Técnicas didácticas. Editorial S\&A. Quito. Ecuador.

Boostrom, R. (2005). The foundation of critical and creative learning in the classroom. New York, Teachers College Press.

Capelástegui Pérez-España, Pilar (2003). Breve Manual para la narración de experiencias innovadoras. Documento en línea: http://www.upch.edu.pe/faedu/documentos/enlaces/guia.pdf (OEI Madrid: Organización de Estados Americanos para la Educación, la Ciencia y la Cultura

Cascón, I. (2000). Análisis de las calificaciones escolares como criterio de rendimiento académico. En red. Recuperado http://www3.usal.es./inico/investigacion/jornadas/jornada2/comunc/cl7.html

Dale H. (2012). Teorías del aprendizaje. Una perspectiva educativa. Pearson Educativa. Sexta edición. México.

Licencia Creative Commons Atribución 4.0 Internacional (CC BY 4.0)

Revista Cátedra, 3(2), pp. 112-125, mayo-agosto 2020. e-ISSN: 2631-2875

https://doi.org/10.29166/10.29166/catedra.v3i2.2072 
De Old, K. (2014). El vacío es materia. Kaosenlared. Recuperado el 2 de marzo de 2020 de: https://kaosenlared.net/el-vacio-es-materia-aproximaci-n-a-la-f-sica-3-edici-n/

Eduteka, Tecnologías de Información y Comunicación para Enseñanza Básica y Media (2013). Taxonomía de Bloom. Recuperado en mayo de 2020, de: http://eduteka.icesi.edu.co/articulos/TaxonomiaBloomCuadro

Gadamer, H. (2007). Análisis de la conciencia efectual. En Verdad y Método. Salamanca, Sígueme.

García, R. (2006). Evaluación del aprendizaje en el nivel universitario: elaboración de exámenes y reactivos objetivos. Recuperado el 17 de junio de 2019 de: www.academia.edu/16517753/Evaluación del aprendizaje en el nivel universitario e laboración de exámenes y reactivos objetivos.

González González, M. T. y Escudero Muñoz, J. M. (1987). Innovación Educativa: teorías y procesos de desarrollo. Barcelona, España: Humanitas.

Hodson, D. (1988). Experiments in science and science teaching, Educational Philosophy y Theory.

Imbernón, F. (1996). En busca del discurso educativo. La escuela, la innovación educativa, el currículo, el maestro y su formación. Buenos Aires, Argentina: Magisterio del Río de la Plata.

Kaosenlared. (2020). Recuperado de: https://kaosenlared.net/colectivo-kaosenlaredquienes-somos/

López, A., Sánchez, H., Espinosa, J. y Camona M. (2013). Elaboración de ítems de opción múltiple. Instituto Nacional de Evaluación Educativa. Quito. Ecuador. https://www.educar.ec/servicios/1-manualelaboracionitems-ineval.pdf

Moreira, M. (2010). ¿Por qué conceptos? ¿Por qué aprendizaje significativo? ¿Por qué actividades colaborativas? ¿Por qué mapas conceptuales? Revista Curriculum 23.

Oviedo, J. (1983). Programa de Formación de Capacitadores Técnico Pedagógico en el Área de planificación Curricular. Pontificia Universidad Católica del Ecuador, Facultad de Ciencias de la Educación. Departamento de pedagogía. Quito. Ecuador.

Perkins, David. (2003). ¿Qué es la comprensión? En: Martha Stone Wiske (Comp.), La enseñanza para la comprensión. Vinculación entre la investigación y la práctica. Trad.: Cristina Piña. Buenos Aires, Argentina: Paidós.

Rojas, R. (2009). Las preguntas y la ciencia escolar: una experiencia con la segunda infancia. Tecne, episteme y didaxis: TEA.

Rua A y Alzate, O. (2012). Las prácticas de laboratorio en la enseñanza de las ciencias natrales. Revista Latinoamericana de Estudios Educativos (Colombia), vol 8.

Sagan, C. (1980). Cosmos. Recuperado el 2 de mayo de 2020 de: http://www.librosmaravillosos.com/Cosmos

Sager, J. (1993). Curso práctico sobre el procesamiento de la terminología. Fundación German Sánchez Ruipérez. Madrid.

Licencia Creative Commons Atribución 4.0 Internacional (CC BY 4.0)

Revista Cátedra, 3(2), pp. 112-125, mayo-agosto 2020. e-ISSN: 2631-2875

https://doi.org/10.29166/10.29166/catedra.v3i2.2072 
Sánchez, I. (2010). Aprendizaje basado en preguntas y su impacto en las estrategias de aprendizaje en Física. X Congreso internacional sobre investigación en didáctica de las Ciencias. Chile.

Szczurek, M. (1989). La estrategia instruccional. Investigación y Posgrado. Recuperado de: https://es.slideshare.net/BelkysGuzman/la-estrategia-instruccional-marioszczurek

Tejada, J. (2012). Los profesores como agentes de la innovación educativa. Recuperado de: https://www.researchgate.net/publication/283350211 Los profesores como agen tes de la innovacion educativa

Torre de la, Saturnino. (1997). Innovación Educativa I. El proceso de innovación. Madrid, España: Dykinson, S. L.

Urbina, I. (2008). Estrategias metodológicas para potenciar la comprensión de la Física. Revista Ingenio, p 27. Colombia.

Young. H. (2009). Física universitaria volumen 1. Decimo segunda edición. Pearson Educación. México.

\section{Autores}

FRANKLIN MOLINA-JIMÉNEZ obtuvo su título de Magíster en Docencia Universitaria y Administración Educativa en la Universidad Tecnológica Indoamérica (Ecuador) en 2011. Obtuvo el título de Licenciado en Ciencias de la Educación, Especialización Matemática y Física en la Universidad Central del Ecuador en 1995.

Colaboró como profesor de Algebra en la Universidad de las Fuerzas Armadas ESPE. Actualmente es profesor titular de Física y Didáctica de la Matemática y Física de la Carrera de Pedagogía de las Ciencias Experimentales Matemática y Física de la Facultad de Filosofía Letras y Ciencias de la Educación de la Universidad Central del Ecuador. Sus principales temas de investigación están orientados a la didáctica de la física y de la geometría plana. Primer lugar en el concurso organizado por la Secretaria de Educación Municipal de Quito y la Fundación Fidal en la categoría Tics aplicadas al proceso de enseñanza aprendizaje, es autor de capítulos de libros de física y geometría plana, ponencias y artículos publicados en varias revistas.

LUCIA GOYES-CHULDE obtuvo su título de Magíster en Docencia Universitaria y Administración Educativa en la Universidad Tecnológica Indoamérica (Ecuador) en 2011. Obtuvo el título de Licenciada en Ciencias de la Educación Especialización Matemática y Física en la Universidad Central del Ecuador en 1995.

Profesora titular de física y matemática en instituciones públicas y privadas y actualmente es profesora de Física de la Unidad Educativa Municipal Calderón. Primer lugar en el concurso organizado por la Secretaria de Educación Municipal de Quito y la Fundación Fidal en la categoría Tics aplicadas al proceso de enseñanza aprendizaje, sus principales temas de investigación están orientados a la didáctica de la Física. 